\title{
Magnetic Interactions for the Electronic Configuration $d^{5}$
}

\author{
T. M. DunN AND WAI-KeE LI \\ Chemistry Department, University of Michigan, Ann Arbor, Michigan
}

(Received 7 December 1966)

\begin{abstract}
Applying Horie's method, the matrix elements of spin-other-orbit interaction for configuration $d^{5}$ have been calculated. The magnetic interaction energy has also been calculated for $\mathrm{Mn}^{++}$by means of perturbation theory. It is found that spin-other-orbit interaction plays a very important role for atoms or ions with half-filled-shell configuration. Spin-spin interaction is also significant in certain terms.
\end{abstract}

\section{INTRODUCTION}

$\mathbf{T}$ THE magnetic interactions for atoms which have a single unfilled shell of electrons outside a number of closed shells can be written ${ }^{1}$

with

$$
\mathfrak{H C}_{\mathrm{m}}=\mathfrak{H C}_{\mathrm{so}}+\mathfrak{H C}_{\mathrm{ss}}
$$

$$
\begin{aligned}
\mathscr{K}_{\mathrm{BO}}=\zeta_{l} \sum_{i} \mathbf{1}_{i} \cdot \mathbf{s}_{i}-\frac{1}{2} \alpha^{2} \sum_{i \neq j}\left(\frac{\mathbf{r}_{i j}}{\boldsymbol{r}_{i j}{ }^{3}} \times \mathbf{p}_{i}\right) \times\left(\mathbf{s}_{i}+2 \mathbf{s}_{j}\right)=\text { spin-orbit interactions, } \\
\mathfrak{H}_{\mathrm{Bs}}=\frac{1}{2} \alpha^{2} \sum_{i \neq j} \frac{1}{r_{i j}{ }^{3}}\left[\mathbf{s}_{i} \cdot \mathbf{s}_{j}-\frac{3\left(\mathbf{s}_{i} \cdot \mathbf{r}_{i j}\right)\left(\mathbf{s}_{j} \cdot \mathbf{r}_{i j}\right)}{r_{i j}{ }^{2}}\right] \\
=\text { spin-spin interaction, }
\end{aligned}
$$

where $\alpha$ is the fine-structure constant and $\zeta_{l}$ is the spin-orbit parameter:

$$
\zeta_{l}=\frac{1}{2} \alpha^{2} \int_{0}^{\infty} r^{-1}(\partial U / \partial r)\left[R_{l}(r)\right]^{2} r^{2} d r,
$$

and the summations run over all electrons in the unfilled shell. If it is assumed that $\mathfrak{H}_{\mathrm{m}}$ is much smaller than the electrostatic interaction, it is possible to calculate the magnetic interaction energy by means of perturbation theory, using $|v S L J M\rangle$ as the zeroth-order wavefunctions. According to Horie ${ }^{2}$ and to Trees, ${ }^{3}$ the matrix elements of $\mathscr{H}_{\mathrm{so}}$ and $\mathfrak{H}_{\mathrm{Bs}}$ for $l^{n}$ configuration are given by

$$
\begin{aligned}
& \left(l^{n_{v} S L J M}\left|\mathfrak{K}_{\mathrm{so}}\right| l^{n} v^{\prime} S^{\prime} L^{\prime} J M\right)=(-1)^{S+L^{\prime}-J} W\left(S L S^{\prime} L^{\prime} ; J 1\right)\left(l^{n} v S L\left\|\mathfrak{K}_{\mathrm{so}}\right\| l^{n} v^{\prime} S^{\prime} L^{\prime}\right), \\
& \left(l^{n_{v} S L J M}{ }^{1} \mathfrak{K}_{\mathrm{sg}} \mid l^{n} v^{\prime} S^{\prime} L^{\prime} J M\right)=(-1)^{S+L^{\prime}-J} W\left(S L S^{\prime} L^{\prime} ; J 2\right)\left(l^{n} v S L\left\|\mathcal{K}_{\mathrm{ss}}\right\| l^{n} v^{\prime} S^{\prime} L^{\prime}\right),
\end{aligned}
$$

where $W(a b c d ; e f)$ is the well-known Racah coefficient ${ }^{4}$ and $v$ is the seniority number also defined by Racah. ${ }^{5}$

The double-barred matrix elements of $\mathfrak{H}_{\mathrm{ss}}$ for $d^{n}$ configurations $(n=2,3,4,5)$ have been calculated by Trees ${ }^{3}$; while those of $\mathfrak{F}_{\mathrm{so}}$ for configurations $d^{2}, d^{3}$, and $d^{4}$ have been reported by Horie. ${ }^{2}$

\section{CALCULATION OF $\left(d^{5} v S L\left\|\mathcal{H}_{\mathrm{Bo}}\right\| d^{5} v^{\prime} S^{\prime} L^{\prime}\right)$}

$\mathcal{F}_{\text {во }}$ can be further divided into two parts, namely, the individual spin-orbit interaction [first term in (2)] and the spin-other-orbit interaction [last term in (2)]. The matrix elements

$$
\left(l^{n_{v}} S L J M\left\|\zeta_{i} \sum_{i} \mathbf{1}_{i} \cdot \mathbf{s}_{i}\right\| l^{n^{\prime}{ }^{\prime}} S^{\prime} L^{\prime} J M\right)
$$

\footnotetext{
1 M. Blume and R. E. Watson, Proc. Roy. Soc. (London) A270, 127 (1962).

2 H. Horie, Progr, Theoret. Phys. (Kyoto) 10, 296 (1953).

R. E. Trees, Phys. Rev. 82, 683 (1951).

${ }^{4}$ G. Racah, Phys. Rev. 62, 438 (1942).

${ }^{5}$ G. Racah, Phys. Rev. 63, 367 (1943).
} 
were first calculated by Racah ${ }^{5}$ by means of the irreducible-tensor-operator method and are given by $\left(l^{n} v S L J M\left|\zeta_{l} \sum_{i} l_{i} \cdot s_{i}\right| l^{n} v^{\prime} S^{\prime} L^{\prime} J M\right)=(-1)^{S+L^{\prime}-J} \zeta_{i}[l(l+1)(2 l+1)]^{1 / 2}\left(l^{n} v S L\left\|V^{(11)}\right\| l^{n} v^{\prime} S^{\prime} L^{\prime}\right) W\left(S L S^{\prime} L^{\prime}, J 1\right)$,

where the elements $\left(l^{n} v S L\left\|V^{(11)}\right\| l^{n} v^{\prime} S^{\prime} L^{\prime}\right)$ are well tabulated in numerous books. ${ }^{6}$ In calculating the doublebarred matrix elements for spin-other-orbit interaction, Horie ${ }^{2}$ found that part of this interaction also behaves as an effective individual spin-orbit interaction. These two similar interactions are then combined together and defined as $\mathfrak{F}_{\mathrm{so}}{ }^{(\mathrm{I})}$. For configuration $d^{n}$, the elements $\left(d^{n_{v}} S L\left\|\mathcal{H}_{\mathrm{so}}{ }^{(\mathrm{I})}\right\| d^{n} v^{\prime} S^{\prime} L^{\prime}\right)$ take the following form:

where

$$
\left(d^{n} v S L\left\|\mathfrak{F}_{\mathrm{so}}^{(\mathbf{I})}\right\| d^{n} v^{\prime} S^{\prime} L^{\prime}\right)=\zeta^{\prime}\left(d^{n} v S L\left\|(30)^{1 / 2} V^{(1)}\right\|_{1}^{\prime} d^{n_{v^{\prime}}} S^{\prime} L^{\prime}\right),
$$

$$
\zeta^{\prime}=\zeta_{d}-7(2 n-3) M_{0}+42 M_{2}
$$

$\mathrm{M}_{0}$ and $\mathrm{M}_{2}$ are the following radial parameters (for equivalent $d$-electron systems only):

$$
\begin{aligned}
& M_{0}=\frac{\alpha^{2}}{28} \iint_{r_{1} \geq r_{1}} \frac{1}{r_{1}^{3}} R_{d}^{2}\left(r_{1}\right) R_{d}^{2}\left(r_{2}\right) r_{1}^{2} r_{2}^{2} d r_{1} d r_{2}, \\
& M_{2}=\frac{\alpha^{2}}{196} \iint_{r_{1} \geq r_{1}} \frac{r_{2}^{2}}{r_{1}^{5}} R_{d}^{2}\left(r_{1}\right) R_{d}^{2}\left(r_{2}\right) r_{1}^{2} r_{2}^{2} d r_{1} d r_{2} .
\end{aligned}
$$

The matrix elements for the remaining part of spin-other-orbit interaction, defined as $\mathfrak{T}_{\mathrm{so}}{ }^{(\mathrm{II})}$, can be obtained as follows [Eq. (19) in Horie's paper]:

$$
\begin{aligned}
& \mathscr{H}_{\mathrm{so}}{ }^{(1 \mathrm{I})}=\mathscr{H}_{\mathrm{so}}-\mathfrak{H}_{\mathrm{so}}{ }^{(1)} \text {, } \\
& \left(l^{n} v S\left\|\mathcal{H}_{\mathrm{so}}^{(\mathrm{II})}\right\| l^{n} v^{\prime} S^{\prime} L^{\prime}\right)=-2 \sqrt{3} \sum_{k, K}\left(l\left\|U^{(K, k)}\right\| l\right)\left(l\left\|C^{(K)}\right\| l\right) \\
& \times\left[\sum_{v^{\prime \prime}, L^{\prime \prime}}\left(l^{n} \mathcal{V} S L\left\|U^{(K)}\right\| l^{n_{\mathcal{V}^{\prime \prime}}} S L^{\prime \prime}\right)\left(l^{n_{\mathcal{V}^{\prime \prime}}} S L^{\prime \prime}\left\|V^{(1 k)}\right\| l^{n_{\mathcal{V}^{\prime}}} S^{\prime} L^{\prime}\right) W\left(L K L^{\prime} k ; L^{\prime \prime} 1\right)\right. \\
& \left.+2 \sum_{\boldsymbol{z}^{\prime \prime}, L^{\prime \prime}}\left(l^{n} v S L\left\|U^{(k)}\right\| l^{n_{v^{\prime \prime}}} S L^{\prime \prime}\right)\left(l^{n_{v^{\prime \prime}}} S L^{\prime \prime}\left\|V^{(1 K)}\right\| l^{n} v^{\prime} S^{\prime} L^{\prime}\right) W\left(L k L^{\prime} K ; L^{\prime \prime} 1\right)\right] M^{k-1} \text {. }
\end{aligned}
$$

For the notation used in (12), see Horie's paper. Expanding (12) for the $d^{n}$ configuration yields

where

$$
\left(l^{n} v S L\left\|\mathfrak{H}_{80}{ }^{(I I)}\right\| l^{n} v^{\prime} S^{\prime} L^{\prime}\right)=(13 \mathrm{a})+(13 \mathrm{~b})+(13 \mathrm{c})+(13 \mathrm{~d})+(13 \mathrm{e})+(13 \mathrm{f})+(13 \mathrm{~g}),
$$

$$
\begin{gathered}
-28[L(L+1)(2 L+1) S(S+1)(2 S+L)]^{1 / 2} M_{0} \delta_{S S^{\prime}} \delta_{L L^{\prime}}, \\
-30(70)^{1 / 2} \sum_{v^{\prime \prime}, L^{\prime \prime}}\left(d^{n} v S L\left\|U^{(2)}\right\| d^{n} v^{\prime \prime} S L^{\prime \prime}\right)\left(d^{n} v^{\prime \prime} S L^{\prime \prime}\left\|V^{(11)}\right\| d^{n} v^{\prime} S^{\prime} L^{\prime}\right) W\left(L 2 L^{\prime} 1 ; L^{\prime \prime} 1\right) M_{0}, \\
-20(21)^{1 / 2}[L(L+1)(2 L+1)]^{1 / 2}\left(d^{n} v S L\left\|V^{(12)}\right\| d^{n} v^{\prime} S^{\prime} L^{\prime}\right) W\left(L 1 L^{\prime} 2 ; L 1\right) M_{0}, \\
-280(105)^{1 / 2} \sum_{v^{\prime \prime}, L^{\prime \prime}}\left(d^{n} v S L\left\|U^{(2)}\right\| d^{n} v^{\prime \prime} S L^{\prime \prime}\right)\left(d^{n} v^{\prime \prime} S L^{\prime \prime}\left\|V^{(13)}\right\| d^{n v^{\prime}} S^{\prime} L^{\prime}\right) W\left(L 2 L^{\prime} 3 ; L^{\prime \prime} 1\right) M_{2}, \\
-560(105)^{1 / 2} \sum_{v^{\prime \prime}, L^{\prime \prime}}\left(d^{n} v S L\left\|U^{(3)}\right\| d^{n} v^{\prime \prime} S L^{\prime \prime}\right)\left(d^{n} v^{\prime \prime} S L^{\prime \prime}\left\|V^{(12)}\right\| d^{n} v^{\prime} S^{\prime} L^{\prime}\right) W\left(L 3 L^{\prime} 2 ; L^{\prime \prime} 1\right) M_{2}, \\
-1260(7)^{1 / 2} \sum_{v^{\prime \prime}, L^{\prime \prime}}\left(d^{n} v S L\left\|U^{(4)}\right\| d^{n} v^{\prime \prime} S L^{\prime \prime}\right)\left(d^{n} v^{\prime \prime} S L^{\prime \prime}\left\|V^{(13)}\right\| d^{n} v^{\prime} S^{\prime} L^{\prime}\right) W\left(L 4 L^{\prime} 3 ; L^{\prime \prime} 1\right) M_{2}, \\
-2520(7)^{1 / 2} \sum_{v^{\prime \prime}, L^{\prime \prime}}\left(d^{n} v S L\left\|U^{(3)}\right\| d^{n} v^{\prime \prime} S L^{\prime \prime}\right)\left(d^{n} v^{\prime \prime} S L^{\prime \prime}\left\|V^{(14)}\right\| d^{n} v^{\prime} S^{\prime} L^{\prime}\right) W\left(L 3 L^{\prime} 4 ; L^{\prime \prime} 1\right) M_{2} .
\end{gathered}
$$

${ }^{6}$ J. C. Slater, Quantum Theory of Atomic Structure (McGraw-Hill Book Co., 1960), Vol. 2. 
The matrix elements $\left(d^{n} v S L\left\|U^{(r)}\right\| d^{n} v^{\prime} S^{\prime} L^{\prime}\right)$ and $\left(d^{n} v S L\left\|V^{(1 r)}\right\| d^{n v^{\prime}} S^{\prime} L^{\prime}\right)$ can be calculated by the method based on the coefficients of fractional percentage. ${ }^{6}$ Applying Formula (13), the matrix elements $\left(d^{5} v S L\left\|\mathscr{H}_{\mathrm{so}}{ }^{(\mathrm{II})}\right\| d^{5} v^{\prime} S^{\prime} L^{\prime}\right)$ can be calculated and the nonvanishing ones are given in Table $\mathrm{I}$.

\section{ANALYSIS OF MULTIPLETS OF $d^{5}$ CONFIGURATION OF $\mathrm{Mn}^{++}$}

The $d^{5}$ configuration of the spectrum of $\mathrm{Mn}^{++}$was chosen for analysis. ${ }^{7}$ It is well known that diagonal matrix elements for individual spin-orbit interaction operator $\left(\zeta_{i} \sum_{i} 1_{i} \cdot s_{i}\right)$ vanish for atoms or ions with half-filled-shell configuration. ${ }^{8}$ Therefore, it might be assumed that the contributions from spin-other-orbit and spin-spin interactions would be more important in

TABLE I. Nonvanishing matrix elements of $\left(d^{5} v S L\left\|\mathfrak{K}_{\mathrm{so}}(\mathbf{n})\right\| d^{5} v^{\prime} S^{\prime} L^{\prime}\right)$.

\begin{tabular}{|c|c|c|}
\hline$v S L$ & $v^{\prime} S^{\prime} L^{\prime}$ & \\
\hline${ }^{4} G$ & ${ }_{45}^{4} G$ & $-10 \sqrt{3}\left(109 M_{0}-52 M_{2}\right)$ \\
\hline${ }_{5}^{4} G$ & $2_{5} G$ & $-(330)^{1 / 2}\left(M_{0}+137 M_{2}\right)$ \\
\hline${ }_{4}^{4} G$ & $2_{5} F$ & $3(30)^{1 / 2}\left(9 M_{0}+113 M_{2}\right)$ \\
\hline $4_{3} F$ & ${ }_{3}^{4} F$ & $-2(35)^{1 / 2}\left(101 M_{0}-8 M_{2}\right)$ \\
\hline $4_{3} F$ & ${ }_{2} G$ & $-15(10)^{1 / 2}\left(3 M_{0}+55 M_{2}\right)$ \\
\hline $4_{3} F$ & ${ }_{2} F$ & $(14)^{1 / 2}\left(19 M_{0}+23 M_{2}\right)$ \\
\hline $4_{3} F$ & ${ }_{3} D$ & $-4 \sqrt{2}\left(4 M_{0}+533 M_{2}\right)$ \\
\hline${ }_{5} D$ & $a_{5} D$ & $-25 \sqrt{2}\left(19 M_{0}-2 M_{2}\right)$ \\
\hline${ }_{5}^{4} D$ & ${ }_{5}^{2} F$ & $16(10)^{1 / 2}\left(M_{0}+67 M_{2}\right)$ \\
\hline${ }_{4}^{4} D$ & $2_{1} D$ & $8(35)^{1 / 2}\left(M_{0}+37 M_{2}\right)$ \\
\hline${ }_{4} D$ & ${ }_{2} D$ & $-2(10)^{1 / 2}\left(19 M_{0}+643 M_{2}\right)$ \\
\hline${ }_{4}^{4} P$ & ${ }_{3}^{4} P$ & $-7(10)^{1 / 2}\left(13 M_{0}-54 M_{2}\right)$ \\
\hline${ }_{3}^{4} P$ & ${ }_{3} D$ & $-12(7)^{1 / 2}\left(M_{0}-23 M_{2}\right)$ \\
\hline${ }_{3} P$ & ${ }_{3}^{2} P$ & $-2(14)^{1 / 2}\left(M_{0}+117 M_{2}\right)$ \\
\hline $2_{5} I$ & $2_{5}^{2} I$ & $-3(91)^{1 / 2}\left(39 M_{0}+68 M_{2}\right)$ \\
\hline$z_{3} H$ & ${ }_{3}^{3} H$ & {$\left[-9(55)^{1 / 2} / 5\right]\left(61 M_{0}+52 M_{2}\right)$} \\
\hline${ }_{3}^{2} H$ & $2_{3} G$ & {$\left[-24(55)^{1 / 2} / 5\right]\left(3 M_{0}-4 M_{2}\right)$} \\
\hline $2_{3} G$ & ${ }_{3}^{2} G$ & {$\left[-9(30)^{x / 2} / 10\right]\left(93 M_{0}-649 M_{2}\right)$} \\
\hline $2_{3} G$ & $2 a$ & {$\left[-3(10)^{1 / 2} / 2\right]\left(9 M_{0}+253 M_{2}\right)$} \\
\hline${ }^{2}{ }_{6} G$ & ${ }_{2}^{2} G$ & {$\left[-(30)^{1 / 2} / 2\right]_{\left(157 M_{0}-901 M_{2}\right)}$} \\
\hline $2_{5} G$ & $2_{5} F$ & {$\left[-3(330)^{1 / 2} / 2\right)\left(3 M_{0}+131 M_{2}\right)$} \\
\hline${ }_{3} F$ & ${ }_{3}^{2} F$ & {$\left[-5(14)^{1 / 2} / 2\right]\left(47 M_{0}+145 M_{2}\right)$} \\
\hline${ }_{3}^{2} F$ & ${ }_{3}^{2} D$ & $44 \sqrt{2}\left(M_{0}+32 M_{2}\right)$ \\
\hline${ }_{3}^{2} F$ & ${ }_{3}^{2} F$ & {$\left[-(14)^{1 / 2} / 2\right]\left(239 M_{0}-307 M_{2}\right)$} \\
\hline${ }_{25} F$ & ${ }_{1}^{2} D$ & $4(70)^{1 / 2}\left(M_{0}+37 M_{2}\right)$ \\
\hline$z_{3} F$ & $2_{5}^{2} D$ & $-16(5)^{1 / 2}\left(M_{0}-38 M_{2}\right)$ \\
\hline$z_{1}^{2} D$ & ${ }_{1}^{2} D$ & $-7(5)^{1 / 2}\left(11 M_{0}+2 M_{2}\right)$ \\
\hline${ }_{2}{ }_{1} D$ & $2_{5} D$ & $-4(70)^{1 / 2}\left(M_{0}+37 M_{2}\right)$ \\
\hline $2_{3} D$ & ${ }_{3}^{2} D$ & $-5(5)^{1 / 2}\left(25 M_{0}+242 M_{2}\right)$ \\
\hline${ }_{3} D$ & ${ }_{3} P$ & $-6 \sqrt{2}\left(2 M_{0}-61 M_{2}\right)$ \\
\hline${ }_{5}^{2} D$ & ${ }_{5}^{8} D$ & $-(5)^{1 / 2}\left(61 M_{0}-1868 M_{2}\right)$ \\
\hline $2_{3} P$ & $2_{3} P$ & $-5\left(13 M_{0}-576 M_{2}\right)$ \\
\hline
\end{tabular}

${ }^{7}$ Alomic Energy Levels, C. E. Moore, Ed. (Natl. Bur. Std. Circ. No. 467,1952 ), Vol. 2 .

${ }^{8}$ E. U. Condon and G. H. Shortley, Theory of Atomic Spectra (Cambridge University Press, Cambridge, England, 1935).
TABLE II. Term values of the $3 d^{5}$ configuration of $\mathrm{Mn}^{+2}\left(\mathrm{~cm}^{-1}\right)$.

\begin{tabular}{|c|c|c|c|}
\hline Term & Obs & Calc & Diff \\
\hline${ }^{6} S$ & 0.0 & 0.0 & \\
\hline${ }_{3}^{4} G$ & 28845.6 & 26845.6 & 0.0 \\
\hline${ }_{3}^{4} P$ & 29200.0 & 29200.0 & 0.0 \\
\hline${ }_{5}^{4} D$ & 32351.2 & 32238.2 & -113.0 \\
\hline $2_{5} I$ & & 39101.0 & \\
\hline${ }_{2}^{2} D$ & & 41303.0 & \\
\hline${ }_{3}^{2} F$ & & 42397.9 & \\
\hline${ }_{4}^{4} F$ & 43617.9 & 43746.9 & 129.0 \\
\hline${ }_{3} H$ & & 46503.8 & \\
\hline${ }_{2}{ }_{6} G$ & & 47575.2 & \\
\hline${ }^{2} F$ & & 50656.7 & \\
\hline${ }_{5}^{2} S$ & & 55279.0 & \\
\hline${ }_{2} D$ & & 61268.0 & \\
\hline${ }_{3}^{2} G$ & & 68754.5 & \\
\hline $2_{3} P$ & & 83047.5 & \\
\hline${ }_{5} D$ & & 89533.1 & \\
\hline
\end{tabular}

a $B=919.94 ; C=3230.12 ; a=74.778$. Mean deviation $= \pm 64.5 \mathrm{~cm}^{-1}$,

determining the component order of a term. In addition, all the terms (ground term and the quartets) which have been observed have lower energy than the ones arising from configurations $3 d^{4} 4 s, 3 d^{4} 4 p$, and so on. (This is in contrast with, for example, the $d^{5}$ configuration of the spectrum of $\mathrm{Cr}^{+}$.) Consequently, configuration interaction may be relatively small. So far none of the doublet terms arising from $d^{5}$ (in $\mathrm{Mn}^{++}$) have been experimentally observed and it is hoped that their position could be accurately estimated from these calculations, thus assisting in any experimental research.

The analysis of the electrostatic interaction energy has been done by Laporte, ${ }^{9}$ and also by Racah. ${ }^{4}$ The formulas for the term values used here have been taken in the same form as was given by Racah. In addition, a correction of the form $a L(L+1)$ has been added to every term. The necessity for this was pointed out first by Trees, ${ }^{10}$ and also by Racah, ${ }^{11}$ and a correction of this form produces greatly improved agreement between theoretical and experimental term values in many spectra of the ion group. The data have been fitted by the method of least squares and the results are given in Table II. The mean deviation is $64.5 \mathrm{~cm}^{-1}$.

Radial parameters $\zeta_{d}, M_{0}$, and $M_{2}$ for $\mathrm{Mn}^{++}$have been evaluated analytically using nonrelativistic Hartree-Fock radial functions by Blume and Watson. ${ }^{2}$ These values have been used here in solving the follow. ing secular determinant:

$$
\begin{aligned}
\operatorname{det} \mid\left(d^{5} v S L I M\left|\pi C_{m}\right| d^{5} v^{\prime} S^{\prime} L^{\prime} J^{\prime} M^{\prime}\right) \\
\quad-(E-E(v, S, L)) \delta_{v v^{\prime}} \delta_{S S^{\prime}} \delta_{L L^{\prime}} \delta_{J J^{\prime}} \delta_{M M^{\prime}} \mid=0,
\end{aligned}
$$

9 O. Laporte, Phys. Rev. 61, 302 (1942).

10. R. E. Trees, Phys. Rev. 85, 382 (1952).

${ }^{11}$ G. Racah, Phys. Rev. 85, 381 (1952).

${ }_{12} \mathrm{M}$. Blume and R. E. Watson, Proc. Roy, Soc. (London) A271, 565 (1963). 
TABLE III. Analysis of the $d^{5}$ configuration of the spectrum of $\mathrm{Mn}^{++}\left(\mathrm{cm}^{-1}\right)$.

\begin{tabular}{|c|c|c|c|c|c|c|c|c|c|}
\hline Term & $J$ & $\begin{array}{l}\text { Experi- } \\
\text { mental } \\
\text { value }\end{array}$ & $\begin{array}{c}\text { After } \\
\text { Individual } \\
\text { spin-orbit } \\
\text { interaction } \\
E(1)\end{array}$ & $\underset{\text { term value } \mid}{\mid E(1)-}$ & $\begin{array}{c}\text { After } \\
\text { complete } \\
\text { spin-orbit } \\
\text { interaction } \\
E(2)\end{array}$ & $\underset{E(1) \mid}{\mid E(2)}$ & $\begin{array}{c}\text { After } \\
\text { complete } \\
\text { magnetic } \\
\text { interactions } \\
E(3)\end{array}$ & $\mid \begin{array}{l}E(3)- \\
E(2) \mid\end{array}$ & $\begin{array}{c}\mid E(3)- \\
\text { experi- } \\
\text { mental } \\
\text { value } \mid\end{array}$ \\
\hline $6_{5} S$ & $2 \frac{1}{2}$ & 0.0 & -20.0 & 20.0 & -19.0 & 1.0 & -19.0 & 1.0 & 19.0 \\
\hline${ }^{4} G$ & $\begin{array}{l}5 \frac{1}{2} \\
4 \frac{1}{2} \\
3 \frac{1}{2} \\
2 \frac{1}{2}\end{array}$ & $\begin{array}{ll}26 & 823.8 \\
26 & 850.9 \\
26 & 859.1 \\
26 & 856.0\end{array}$ & $\begin{array}{ll}26 & 827.7 \\
26 & 833.4 \\
26 & 821.3 \\
26 & 801.5\end{array}$ & $\begin{array}{l}17.9 \\
12.2 \\
24.3 \\
44.1\end{array}$ & $\begin{array}{ll}26 & 785.8 \\
26 & 830.5 \\
26 & 851.2 \\
26 & 875.5\end{array}$ & $\begin{array}{r}41.9 \\
2.9 \\
29.9 \\
74.0\end{array}$ & $\begin{array}{ll}26 & 785.8 \\
26 & 830.4 \\
26 & 851.1 \\
26 & 857.6\end{array}$ & $\begin{array}{r}0.0 \\
0.1 \\
0.1 \\
17.9\end{array}$ & $\begin{array}{r}38.0 \\
20.5 \\
8.0 \\
1.6\end{array}$ \\
\hline${ }_{5}^{4} P$ & $\begin{array}{r}2 \frac{1}{2} \\
1 \frac{1}{2} \\
\frac{1}{2}\end{array}$ & $\begin{array}{ll}29 & 168.2 \\
29 & 241.8 \\
29 & 207.6^{b}\end{array}$ & $\begin{array}{ll}29 & 094.7 \\
29 & 125.9 \\
29 & 168.1\end{array}$ & $\begin{array}{r}105.3 \\
74.1 \\
31.9\end{array}$ & $\begin{array}{ll}29 & 094.2 \\
29 & 134.3 \\
29 & 180.4\end{array}$ & $\begin{array}{r}0.5 \\
8.4 \\
12.3\end{array}$ & $\begin{array}{ll}29 & 093.6 \\
29 & 136.2 \\
29 & 178.1\end{array}$ & $\begin{array}{l}0.6 \\
1.9 \\
2.3\end{array}$ & $\begin{array}{r}74.6 \\
105.6 \\
29.5\end{array}$ \\
\hline${ }_{4}^{4} D$ & $\begin{array}{r}3 \frac{1}{2} \\
2 \frac{1}{2} \\
1 \frac{1}{2} \\
\frac{1}{2}\end{array}$ & $\begin{array}{ll}32 & 307.4 \\
32 & 383.6 \\
32 & 384.0^{b}\end{array}$ & $\begin{array}{ll}32 & 320.4 \\
32 & 407.8 \\
32 & 402.0 \\
32 & 371.1\end{array}$ & $\begin{array}{l}30.8 \\
56.6 \\
50.8 \\
19.9\end{array}$ & $\begin{array}{ll}32 & 302.8 \\
32 & 407.9 \\
32 & 418.4 \\
32 & 398.9\end{array}$ & $\begin{array}{r}17.6 \\
0.1 \\
16.4 \\
27.8\end{array}$ & $\begin{array}{ll}32 & 304.7 \\
32 & 403.2 \\
32 & 418.4 \\
32 & 405.5\end{array}$ & $\begin{array}{l}1.9 \\
4.7 \\
0.0 \\
6.6\end{array}$ & $\begin{array}{r}2.7 \\
4.6 \\
34.4\end{array}$ \\
\hline${ }_{5}^{2} I$ & $\begin{array}{l}6 \frac{1}{2} \\
5 \frac{1}{2}\end{array}$ & & $\begin{array}{ll}39 & 101.0 \\
39 & 050.2\end{array}$ & $\begin{array}{c}0 \\
50.8\end{array}$ & $\begin{array}{ll}39 & 101.0 \\
39 & 084.3\end{array}$ & $\begin{array}{c}0 \\
34.1\end{array}$ & $\begin{array}{ll}39 & 101.0 \\
39 & 084.3\end{array}$ & $\begin{array}{l}0.0 \\
0.0\end{array}$ & \\
\hline${ }^{2} D$ & $\begin{array}{l}2 \frac{1}{2} \\
1 \frac{1}{2}\end{array}$ & & $\begin{array}{ll}41 & 017.7 \\
41 & 161.7\end{array}$ & $\begin{array}{l}285.3 \\
141.3\end{array}$ & $\begin{array}{ll}41 & 029.3 \\
41 & 178.7\end{array}$ & $\begin{array}{l}11.6 \\
17.0\end{array}$ & $\begin{array}{ll}41 & 029.4 \\
41 & 178.5\end{array}$ & $\begin{array}{l}0.1 \\
0.2\end{array}$ & \\
\hline${ }_{3}^{2} F$ & $\begin{array}{l}3 \frac{1}{2} \\
2 \frac{1}{2}\end{array}$ & & $\begin{array}{l}42405.8 \\
42665.4\end{array}$ & $\begin{array}{r}7.9 \\
267.5\end{array}$ & $\begin{array}{l}42390.6 \\
42670.3\end{array}$ & $\begin{array}{r}15.2 \\
4.9\end{array}$ & $\begin{array}{ll}42 & 390.5 \\
42 & 670.6\end{array}$ & $\begin{array}{l}0.2 \\
0.3\end{array}$ & \\
\hline${ }_{3}^{4} F$ & $\begin{array}{l}4 \frac{1}{2} \\
3 \frac{1}{2} \\
2 \frac{1}{2} \\
1 \frac{1}{2}\end{array}$ & $\begin{array}{ll}43 & 571.7 \\
43 & 601.6 \\
43 & 667.9 \\
43 & 674.1\end{array}$ & $\begin{array}{ll}43 & 570.1 \\
43 & 600.1 \\
43 & 685.8 \\
43 & 758.6\end{array}$ & $\begin{array}{r}47.8 \\
17.8 \\
67.9 \\
140.7\end{array}$ & $\begin{array}{ll}43 & 542.1 \\
43 & 601.1 \\
43 & 705.1 \\
43 & 790.7\end{array}$ & $\begin{array}{r}28.0 \\
1.0 \\
19.3 \\
32.1\end{array}$ & $\begin{array}{ll}43 & 540.9 \\
43 & 603.7 \\
43 & 705.5 \\
43 & 787.9\end{array}$ & $\begin{array}{l}1.2 \\
2.6 \\
0.4 \\
2.8\end{array}$ & $\begin{array}{r}30.8 \\
2.1 \\
37.6 \\
113.8\end{array}$ \\
\hline${ }_{3}^{2} H$ & $\begin{array}{l}5 \frac{1}{2} \\
4 \frac{1}{2}\end{array}$ & & $\begin{array}{ll}46 & 572.4 \\
46 & 362.6\end{array}$ & $\begin{array}{r}68.6 \\
141.2\end{array}$ & $\begin{array}{ll}46 & 549.1 \\
46 & 389.9\end{array}$ & $\begin{array}{l}23.3 \\
27.3\end{array}$ & $\begin{array}{ll}46 & 549.1 \\
46 & 389.8\end{array}$ & $\begin{array}{l}0.0 \\
0.0\end{array}$ & \\
\hline $2_{5} G$ & $\begin{array}{l}4 \frac{1}{2} \\
3 \frac{1}{2}\end{array}$ & & $\begin{array}{ll}47 & 771.3 \\
47 & 597.5\end{array}$ & $\begin{array}{r}196.1 \\
22.3\end{array}$ & $\begin{array}{l}47758.8 \\
47603.5\end{array}$ & $\begin{array}{r}12.5 \\
6.0\end{array}$ & $\begin{array}{ll}47 & 758.8 \\
47 & 603.3\end{array}$ & $\begin{array}{l}0.0 \\
0.2\end{array}$ & \\
\hline $2_{\mathfrak{b}} F$ & $\begin{array}{l}3 \frac{1}{2} \\
2 \frac{1}{2}\end{array}$ & & $\begin{array}{ll}50 & 681.3 \\
50 & 663.2\end{array}$ & $\begin{array}{r}24.6 \\
6.5\end{array}$ & $\begin{array}{ll}50 & 669.3 \\
50 & 677.5\end{array}$ & $\begin{array}{l}12.0 \\
14.3\end{array}$ & $\begin{array}{ll}50 & 669.3 \\
50 & 677.6\end{array}$ & $\begin{array}{l}0.0 \\
0.1\end{array}$ & \\
\hline${ }_{2}^{2} S$ & $\frac{1}{2}$ & & 55278.3 & 0.7 & $55 \quad 278.3$ & 0.0 & 55278.3 & 0.0 & \\
\hline${ }_{3}^{2} D$ & $\begin{array}{l}2 \frac{1}{2} \\
1 \frac{1}{2}\end{array}$ & & $\begin{array}{ll}61 & 308.7 \\
61 & 284.3\end{array}$ & $\begin{array}{l}40.7 \\
16.3\end{array}$ & $\begin{array}{ll}61 & 291.6 \\
61 & 305.8\end{array}$ & $\begin{array}{l}17.1 \\
21.5\end{array}$ & $\begin{array}{ll}61 & 291.6 \\
61 & 305.8\end{array}$ & $\begin{array}{l}0.0 \\
0.0\end{array}$ & \\
\hline${ }_{3}{ }_{3} G$ & $\begin{array}{l}4 \frac{1}{2} \\
3 \frac{1}{2}\end{array}$ & & $\begin{array}{ll}68 & 759.5 \\
68 & 772.5\end{array}$ & $\begin{array}{r}5.0 \\
18.0\end{array}$ & $\begin{array}{ll}68 & 754.1 \\
68 & 778.0\end{array}$ & $\begin{array}{l}5.4 \\
5.5\end{array}$ & $\begin{array}{ll}68 & 754.1 \\
68 & 778.0\end{array}$ & $\begin{array}{l}0.0 \\
0.0\end{array}$ & \\
\hline${ }_{3} P$ & $\begin{array}{r}1 \frac{1}{2} \\
\frac{1}{2}\end{array}$ & & $\begin{array}{ll}83 & 050.0 \\
83 & 060.2\end{array}$ & $\begin{array}{r}2.5 \\
12.7\end{array}$ & $\begin{array}{ll}83 & 055.1 \\
83 & 049.0\end{array}$ & $\begin{array}{r}5.1 \\
11.2\end{array}$ & $\begin{array}{ll}83 & 055.1 \\
83 & 049.0\end{array}$ & $\begin{array}{l}0.0 \\
0.0\end{array}$ & \\
\hline${ }_{2}{ }_{5} D$ & $\begin{array}{l}2 \frac{1}{2} \\
1 \frac{1}{2}\end{array}$ & & $\begin{array}{ll}89 & 548.5 \\
89 & 538.1\end{array}$ & $\begin{array}{r}15.4 \\
5.0\end{array}$ & $\begin{array}{ll}89 & 553.5 \\
89 & 529.2\end{array}$ & $\begin{array}{l}5.0 \\
8.9\end{array}$ & $\begin{array}{ll}89 & 553.5 \\
89 & 529.2\end{array}$ & $\begin{array}{l}0.0 \\
0.0\end{array}$ & \\
\hline Average & & & & 56.5 & & 15.4 & & 1.2 & 34.9 \\
\hline
\end{tabular}

$\zeta_{\mathrm{I}}=342 \mathrm{~cm}^{-1} ; M_{0}=0.205 \mathrm{~cm}^{-1} ; M_{2}=0.016 \mathrm{~cm}^{-1}$.

$\mathrm{b}$ These levels have been observed by $O$. Riquelme (private communication).

where $E(\eta, S, L)$ are the term values. Experimental term values have been used for the quartets.

Since the selection rules for $\mathfrak{H}_{m}$ are $\Delta J, \Delta M=0$, the above determinant can be broken up into six smaller determinants, namely, $J=\frac{1}{2}(4 \times 4), J=1 \frac{1}{2}(7 \times 7), J=$ $2 \frac{1}{2}(10 \times 10), \quad J=3 \frac{1}{2}(7 \times 7), \quad J=4 \frac{1}{2}(5 \times 5)$, and $J=$ $5 \frac{1}{2}(3 \times 3)$. The results are tabulated in Table III.
From Table III it can be seen that the average individual spin-orbit and spin-other-orbit contributions are 56.5 and $15.4 \mathrm{~cm}^{-1}$, respectively. This indicates that, for atoms or ions with half-filled-shell configuration, spin-other-orbit interaction plays a rather important role in explaining the multiplets ordering, as observed from their atomic spectra. Even though the 
average spin-spin contribution is only $1.2 \mathrm{~cm}^{-1}$, it could be significant in certain terms (e.g., the quartet terms of $d^{5}$ ).

The major sources of error in the calculation probably occur by virture of the fact that configuration interaction has been completely ignored. The radial parameters used here were calculated using the Hartree-Fock wavefunction tabulated by Blume and Watson. These values might well be in error since correlation energy is neglected. However, the relative contributions from different interactions should be accurate. Also the matrix elements may be in error owing to the error in the choice of basis wavefunctions.

\section{ACKNOWLEDGMENT}

The authors wish to thank Dr. S. M. Blinder for many helpful discussions.

THE JOURNAL OF CHEMICAL PHYSICS

VOL UM E 46, N UM B E R 8

15 A P R I L 1967

\title{
Generalized Oscillator Strengths and Absolute-Zero-Angle Cross Sections for Inelastic Electron-Helium Collisions
}

\author{
H. G. M. Heideman and L. VRIENS \\ Physics Laboratory of the University of Utrecht, The Netherlands
}

(Received 6 July 1966)

\begin{abstract}
Absolute-zero-angle cross sections for excitation of helium to the $2^{1} P$ level have been calculated with the Bethe theory, using Lassettre's generalized oscillator strengths (for $1^{1} S \rightarrow 2^{1} P$ ) normalized to the optical oscillator strength of Schiff and Pekeris. Absolute-zero-angle cross sections (in the 25-1000 eV energy region) for transitions to several other helium states were obtained, using (a) the calculated cross section for excitation to the $2^{1} P$ level and (b) already measured cross-section ratios (obtained from energy-loss spectra) or known optical oscillator strengths. These cross sections, which are not very accurate for small incident electron energies due to application of the Bethe theory, have been used to determine generalized oscillator strengths for the transitions $1^{1} S \rightarrow 3^{1} P, 4^{1} P, 5^{1} P, 6^{1} P, 2^{1} S$, and $3^{1} S$. It is pointed out that in the calculation of generalized oscillator strengths possible errors in the zero-angle cross sections used may cancel out, which is illustrated for the transition $1^{1} S \rightarrow 3^{1} P$. "Quadrupole" matrix elements have been derived for the $1^{1} S \rightarrow 2^{1} S, 3^{1} S$ transitions. In most cases the agreement with both experimental and theoretical results of other investigations is very good.
\end{abstract}

\section{INTRODUCTION}

$\mathbf{F}$ IOR the past 35 years measurements and calculations on excitation cross sections for electronhelium collisions have been carried out by various investigators. In the early thirties Whiddington and Woodroofe, ${ }^{1}$ Womer, ${ }^{2}$ and van Atta $^{3}$ measured impact spectra of several gases, yielding among other things ratios of zero-angle scattering cross sections. Their experiments, however, were severely limited by a lack of energy resolution and poorly defined collection geometries. With their apparatus it was not possible, for instance, to separate the $2^{1} S$ and $2^{1} P$ states of helium, and the change of collection geometry did not allow satisfactory determination of the energy dependences of the cross-section ratios. The development of high-resolution electron spectrometers has considerably enhanced the state of affairs. Silverman and Lassettre ${ }^{4}$ recently measured the electron-impact spec-

\footnotetext{
${ }^{1}$ R. Widdington and E. G. Woodroofe, Phil. Mag. 20, 1109 (1935).

2 R. L. Womer, Phys. Rev. 45, 689 (1934).

${ }^{3}$ L. C. Van Atta, Phys. Rev. 38, 876 (1931).

${ }_{4}$ S. M. Silverman and E. N. Lassettre, J. Chem. Phys. 40, 1265 (1964).
}

trum of helium at energies higher than $200 \mathrm{eV}$ and for different scattering angles. From this data they deduced for several transitions in helium generalized oscillator strengths as a function of momentum transfer. It is known that these oscillator strength curves measured at relatively high energy are of great importance and may be used, for instance, to calculate total cross sections.

Electron-impact spectra of helium, providing zeroangle cross sections for several discrete transitions relative to the $1^{1} S \rightarrow 2^{1} P$ transition, have been presented $d^{5}$ at the Fourth International Conference on the Physics of Electronic and Atomic Collisions. These measurements covered an incident energy region from $80 \mathrm{eV}$ down to near threshold. The low incident energies also made it possible to observe the triplet excitations, which only have significant cross sections close to threshold. The energy resolution of the apparatus ${ }^{6}$ was better than $0.1 \mathrm{eV}$, which is good enough to completely separate all $n=2$ states as well as the $3^{3} S$ and $3^{1} S$

${ }^{5}$ G. E. Chamberlain, H. G. M. Heideman, J. A. Simpson, and C. E. Kuyatt, Proc. Intern. Conf. Phys. Electron. At. Collisions 4th, 1965, Quebec, Canada (1965).

'J. A. Simpson, Rev. Sci. Instr. 35, 1698 (1964). 